\title{
Acceptability and Feasibility of a Guided Biopsychosocial Online Intervention for Cancer Patients Undergoing Chemotherapy
}

\author{
Miriam Grapp $^{1,2}$ (D) Friederike Rosenberger ${ }^{3}$ (D) Elena Hemlein ${ }^{4}$ (D) Eva Klein $^{5}$ (D) Hans-Christoph Friederich $^{1}$ (D) \\ Imad Maatouk ${ }^{1,2}$ (D)
}

Published online: 18 June 2020

(C) The Author(s) 2020

\begin{abstract}
Chemotherapy is a physically and psychologically highly demanding treatment, and specific Internet-based interventions for cancer patients addressing both physical side effects and emotional distress during chemotherapy are scarce. This study examined the feasibility and acceptability of a guided biopsychosocial online intervention for cancer patients undergoing chemotherapy $(\mathrm{OPaCT})$. A pre-post, within-participant comparison, mixed-methods research design was followed. Patients starting chemotherapy at the outpatient clinic of the National Center for Tumor Diseases in Heidelberg, Germany, were enrolled. Feasibility and acceptability were evaluated through intervention uptake, attrition, adherence and participant satisfaction. As secondary outcomes, PHQ9, GAD-7, SCNS-SF34-G and CBI-B-D were administered. A total of $N=46$ patients participated in the study (female 76.1\%). The age of participants ranged from 29 to 70 years $(M=49.3, \mathrm{SD}=11.3)$. The most prevalent tumour diseases were breast $(45.7 \%)$, pancreatic $(19.6 \%)$, ovarian $(13.1 \%)$ and prostate cancer $(10.8 \%)$. A total of $N=37$ patients $(80.4 \%)$ completed the OPaCT intervention. Qualitative and quantitative data showed a high degree of participant satisfaction. Significant improvements in the SCNS-SF34 subscale 'psychological needs' were found. Study results demonstrate the feasibility and acceptability of the intervention. The results show that $\mathrm{OPaCT}$ can be implemented well, both in the treatment process and in participants' everyday lives. Although it is premature to make any determination regarding the efficacy of the intervention tested in this feasibility study, these results suggest that $\mathrm{OPaCT}$ has the potential to reduce unmet psychological care needs of patients undergoing chemotherapy.
\end{abstract}

Keywords Chemotherapy $\cdot$ Feasibility $\cdot$ Guided intervention $\cdot$ Online intervention $\cdot$ Supportive care needs

Electronic supplementary material The online version of this article (https://doi.org/10.1007/s13187-020-01792-4) contains supplementary material, which is available to authorized users.

Imad Maatouk

Imad.Maatouk@med.uni-heidelberg.de

1 Department of General Internal and Psychosomatic Medicine, University Hospital Heidelberg, Im Neuenheimer Feld 410, 69120 Heidelberg, Germany

2 Psycho-oncology Service, National Center for Tumor Diseases (NCT), University Hospital Heidelberg, Im Neuenheimer Feld 460, 69120 Heidelberg, Germany

3 Working Group Exercise Oncology, Department of Medical Oncology, National Center for Tumor Diseases (NCT), Im Neuenheimer Feld 460, 69120 Heidelberg, Germany

4 Social Service, National Center for Tumor Diseases (NCT), University Hospital Heidelberg, Im Neuenheimer Feld 460, 69120 Heidelberg, Germany

5 Nursing Service, National Center for Tumor Diseases (NCT), University Hospital Heidelberg, Im Neuenheimer Feld 460, 69120 Heidelberg, Germany

\section{Introduction}

Despite significant advances in modern medicine and biomedical science, chemotherapy remains a physically and psychologically highly demanding treatment. Research has consistently found that 30 to $40 \%$ of people newly diagnosed with cancer experience a marked degree of psychological distress, including clinically significant depressive or anxiety disorders $[1,2]$. Irrespective of cancer type, distress often peaks shortly after cancer diagnosis and during the first 12 months after diagnosis, when a range of medical treatments take place [3]. Psycho-oncology interventions have proven effective in reducing distress, anxiety and depression, and increasing quality of life [4]. However, a high proportion of cancer patients with high levels of psychological distress do not engage with psycho-oncological interventions $[5,6]$. Reasons for this include geographical distance from providers, physical limitations or reduced mobility, patients' preference for managing their emotional and psychological difficulties on their own or stigma about seeking psychological support [7]. 
A growing body of studies concerning eHealth applications and Internet-based interventions (IBIs) have appeared over the last decade, aimed at improving access to psychosocial support for cancer patients [8,9]. IBIs in psycho-oncological care vary widely regarding target group and thematic focus, with IBIs focusing on cancer survivors [10], patients with a specific tumour type [11-14], or on specific issues such as sexual functioning [15], insomnia [16] or fatigue [17]. Recently developed eHealth applications and IBIs for patients undergoing chemotherapy have yielded promising results with regard to dealing with chemotherapy-related symptoms (nausea, vomiting, fatigue, hand-foot syndrome etc.) [18-21] or stress management [22].

Although recent studies indicate that cancer patients have multifarious unmet supportive care needs that occur particularly during the neoadjuvant or adjuvant treatment phase [23, 24], this aspect is only marginally addressed in these interventions. Highest demand for support was observed in psychological needs followed by physical and daily living needs as well as health system and information needs [25]. Therefore, we developed a guided biopsychosocial online intervention for cancer patients undergoing chemotherapy (OPaCT). $\mathrm{OPaCT}$ is based on the 'Supportive Care Framework for Cancer Care' [26]. The intervention is intended to provide orientation and support in dealing with both physical strains and emotional distress, and is aimed at reducing unmet supportive care needs of patients undergoing chemotherapy. The aim of the present study was to investigate the feasibility and acceptability of the OPaCT intervention.

\section{Methods}

\section{Study Design}

We used a pre-post, within-participant comparison, mixedmethods design with pre- and post-treatment questionnaires and semi-structured interviews. The intervention ran from November 2017 to October 2018. The study was conducted in accordance with the Declaration of Helsinki, and the Ethics Committee of the University of Heidelberg (S-320/2017) granted ethical approval. The study was registered at the German Clinical Trials Register (register number: DRKS00013237).

\section{Participants}

German-speaking cancer patients aged 18 years or older, and who started their chemotherapy in the outpatient clinic of the National Center for Tumor Diseases (NCT) in Heidelberg, Germany, were eligible to participate in this study. Participation was independent of stage and severity of tumour disease. Cancer patients with severe psychiatric or cognitive disorders were excluded.

\section{Recruitment and Procedures}

From November 2017 to January 2018, all patients who started chemotherapy at the NCT were informed of the study via flyers, posters and the NCT website. Patients who met the inclusion criteria and gave their written informed consent were included. From February 2018 to July 2018, eligible NCT patients were personally approached by study team members. Patients who agreed to participate in the study were informed about the study goals and procedures and subsequently provided their written informed consent. Reasons for non-participation were documented. After completing a pretreatment questionnaire $\left(T_{0}\right)$, patients received an email with access to the OPaCT programme. Upon completion of the intervention (or dropping out), a post-treatment questionnaire as well as a semi-structured interview was conducted $\left(T_{1}\right)$.

\section{Intervention}

OPaCT is built on the theoretical concept of supportive care needs [26]. To cover the broad range of issues relevant to patients undergoing chemotherapy, OPaCT has been codeveloped by psychologists, physicians, nurses, social workers and sports scientists. OPaCT comprises psychoeducative as well as interactive supporting elements, daily exercises such as mindfulness and guided imagery exercises or diaries and free-text fields for patients to write down their experiences and feelings (self-reflections). Some illustrations of the visual design of OPaCT and layout-exemplary screenshots are provided in Online Resource 1. The programme is designed as an easily accessible, module-based intervention consisting of eight modules which have to be worked through sequentially. Modules focus on physical and psychosocial issues during chemotherapy (Fig. 1) and are designed to take approximately an hour to complete, depending on level of engagement. A psycho-oncologist, trained in the therapeutic use of electronic media, provided supportive guidance and individualized feedback after completion of each module. Individualized feedback to the patients was provided within 1 to max. 5 days after completion of the module. Feedback referred directly to what the patients wrote in the respective module. Additionally, patients had the opportunity to contact the psycho-oncologist via the messenger of $\mathrm{OPaCT}$ at any time.

\section{Measures}

\section{Sample Characteristics}

Sociodemographic information (sex and age) and the patients' clinical characteristics were obtained from the clinic's patient documentation system, which included cancer diagnosis and metastasized cancer (yes/no). 
Fig. 1 Overview of the $\mathrm{OPaCT}$ intervention
Contents:

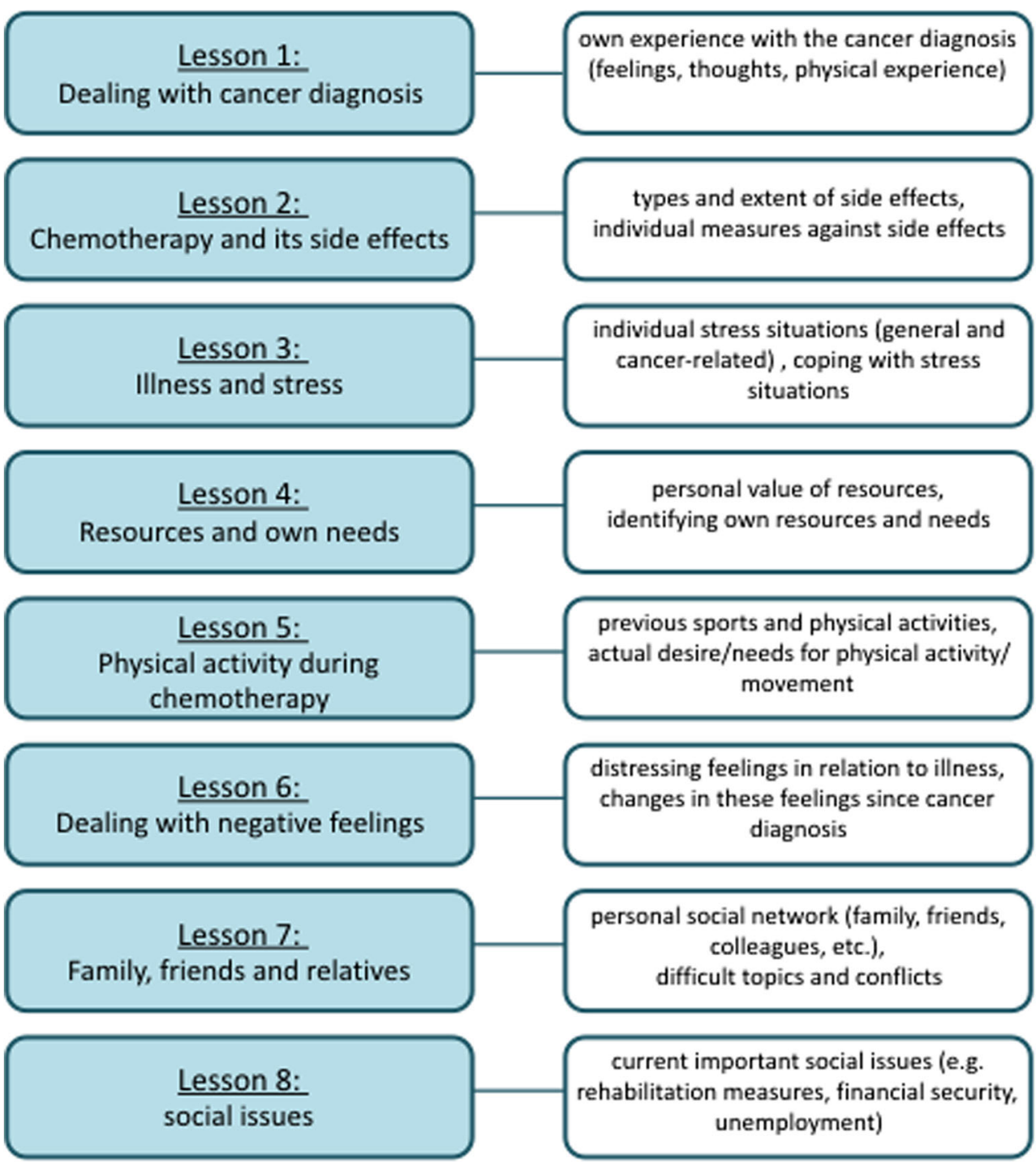

\section{Primary Outcomes: Feasibility and Acceptability}

Feasibility and acceptability were evaluated through intervention uptake, attrition and adherence as well as participant satisfaction. Uptake was assessed as the proportion of patients who agreed to participate in the intervention. Attrition was assessed by the number of patients who dropped out before completing the programme. Adherence refers to the extent to which the patients engaged with the web-based intervention and was operationalized as number of modules completed and the extent to which participants used the additional elements of OPaCT. Participant satisfaction was assessed at postintervention $\left(T_{1}\right)$ via an author-generated questionnaire containing four Likert-type scales on the following items: (1) 'OPaCT was helpful to me', (2) 'OPaCT provided me with new information', (3) 'OPaCT provided practical motivations for everyday life' and (4) 'OPaCT could be well integrated into everyday life'. At $T_{1}$, the study researcher conducted semi-structured interviews to understand personal experiences as well as potential difficulties with the programme. The interview took approximately $30 \mathrm{~min}$.

\section{Secondary Outcomes}

At baseline $\left(T_{0}\right)$ and post-intervention $\left(T_{1}\right)$, the following questionnaires were administered: Depressive syndromes and general anxiety disorder were assessed with the German versions of the Patient Health Questionnaire-9 (PHQ-9) and Generalized Anxiety Disorder (GAD-7) [27]. Supportive care needs were assessed with the German version of the ShortForm Supportive Care Needs Survey Questionnaire (SCNSSF34-G) [28],which is a 34-item self-report questionnaire measuring patients' perceived type and magnitude of need for support in five domains: health system and information, 
psychological, physical and daily living, patient care and support, and sexuality needs. Self-efficacy for coping with cancer was measured using the German version of the brief form of the Cancer Behavior Inventory (CBI-B-D) [29], which consists of 14 items that describe coping behaviours in the context of cancer (maintenance of independence, participation in treatment decision, stress management and affect management).

\section{Data Analysis}

Statistical analyses were performed with IBM SPSS (version 25) software. Descriptive statistics were obtained for participant demographic, disease and treatment characteristics as well as uptake, attrition, adherence and satisfaction profiles. Reasons for non-participation and attrition were collected. Group differences between participants versus nonparticipants and completers versus non-completers were analyzed using $t$ tests, respectively Mann-Whitney $U$ tests for continuous variables, $\chi^{2}$ tests of independence and Fisher's exact test for categorical variables. The sample was not powered to detect significance in the outcome measures; nevertheless, we present non-parametric data in relation to distress (depression and anxiety), supportive care needs and selfefficacy to aid understanding of the potential effect of the intervention within this sample and provide data on which a power calculation for a larger study of efficacy can be based. The Bonferroni-Holm method [30] was used to counteract the problem of multiple comparisons.

\section{Results}

\section{Uptake}

From November 2017 to January 2018, eight patients who started chemotherapy at the NCT expressed interest in participating in the study. Of these patients, four (50\%) could be included in the study. Reasons for exclusion were already completed chemotherapy $(n=3)$ and divergent expectations of participants $(n=1)$. From February 2018 to July 2018, 176 eligible new NCT patients were personally approached by study team members and informed about the OPaCT study, of whom $42(23.9 \%)$ consented to take part. This resulted in an overall response rate of $25 \%$ (46/184). The most common reasons reported for non-participation were too poor physical condition $(n=35)$, no need for psychosocial support $(n=30)$, sense of overwhelming demands $(n=24)$ and concerns about additional involvement with the disease $(n=12)$ (Fig. 2).

\section{Sample Characteristics}

The mean age of participants was 49.2 years (range 29 to 70 years, median 50.5 years). The majority $(76.1 \%)$ of participants were female. Significant differences in age and gender were found between participants and non-participants: nonparticipants were significantly older (mean 56.7 years, range 28 to 81 years, median 56.0 years) than participants, and the proportion of women within the non-participants was significantly lower $(52.2 \%)$. The proportion of patients with metastatic tumour disease was slightly less than one-third among both participants (30.4\%) and non-participants (31.9\%). There were apparent differences between participants and non-participants with regard to cancer type: the most common tumour diseases among participants were breast (45.7\%), pancreatic (19.6\%), ovarian $(13.1 \%)$ and prostate cancer $(10.87 \%)$. The most common tumour diseases among non-participants were breast (34.1\%), stomach/oesophagus (12.7\%), skin (8.7\%), and head and neck cancer $(8.2 \%)$ (Table 1).

\section{Attrition}

Of the 46 patients registered for the intervention, three (6.6\%) never started. Another six (13.3\%) completed only one to three lessons, and 37 (80.4\%) carried out a substantial part of the intervention (six to eight lessons) (Fig. 2). Reasons reported for dropping out were technical difficulties $(n=3)$, aggravation of the physical condition $(n=2)$, lack of time $(n=$ $2)$ and not specified $(n=2)$. We compared demographic and disease characteristics as well as the baseline scores of the 37 patients who completed at least six of eight lessons of the intervention ('completers') with the nine patients who did not complete the intervention ('non-completers'). No differences were found within sex, age and disease-related variables (time since diagnosis and metastasised condition). There were no differences between completers and non-completers with regard to GAD-7, SCNS-SF34-G and CBI-B-D baseline scores. Only depressive symptoms at $T_{0}$ were significantly higher for completers than for non-completers (PHQ-9: $z=$ $-2.197, p=0.027)$.

\section{Adherence}

Over $80 \%$ of participants completed a large part of the intervention (six out of eight lessons). Two-thirds $(67.39 \%)$ of participants carried out the full intervention with all eight consecutive lessons. The majority of participants used the OPaCT element self-reflection $(86.96 \%)$ and the relaxation exercises (82.61\%). About two-thirds of participants (65.22\%) regularly communicated via the integrated email function, whereas only a small proportion used the two diaries $(34.78 \%$ and $26.09 \%$, respectively).

\section{Participant Satisfaction}

The post-treatment questionnaire was returned by 36 participants (33 completers and three non-completers). 
Fig. 2 Flow diagram of the uptake and attrition of the $\mathrm{OPaCT}$ intervention

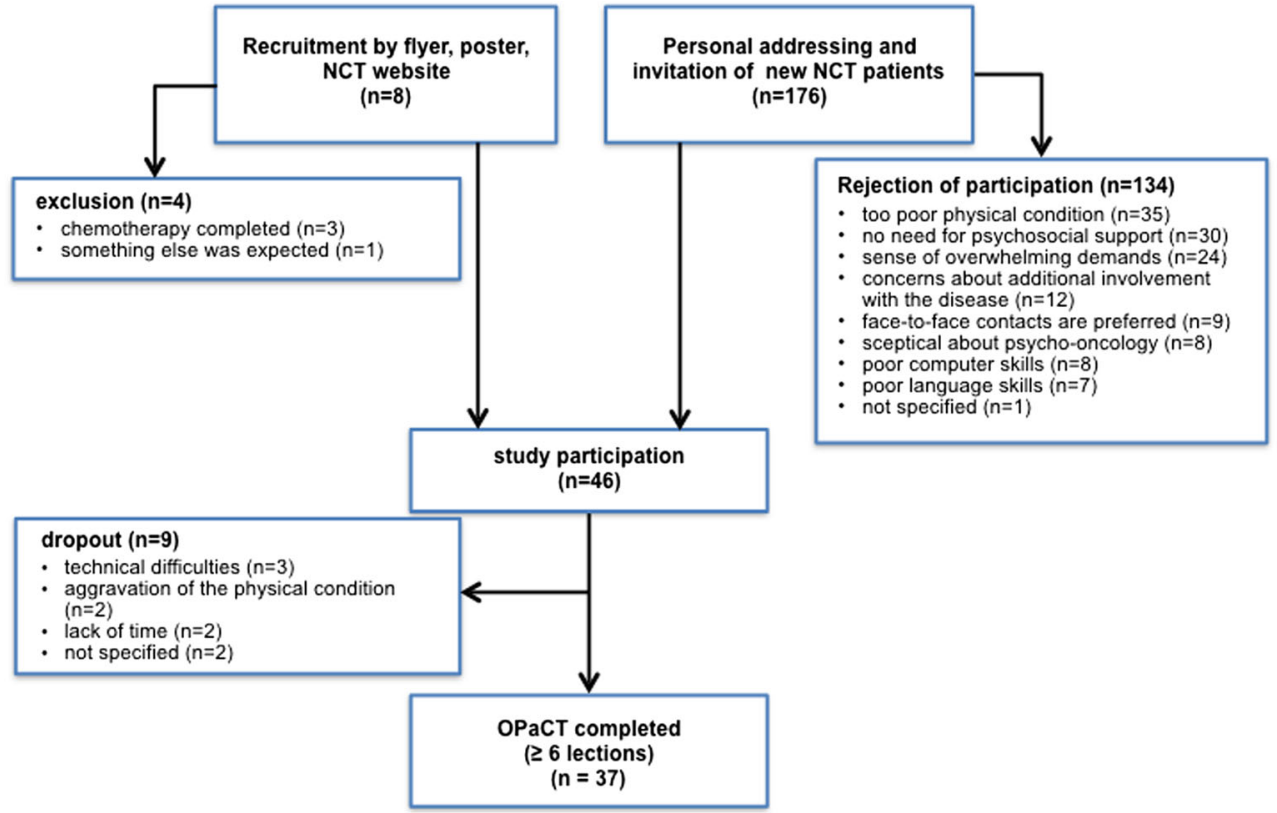

Questionnaire responses indicated the majority of patients experienced OPaCT as helpful $(86.11 \%)$. Most patients found that $\mathrm{OPaCT}$ provided practical motivations for everyday life $(83.33 \%)$ and that it could be well integrated into everyday life $(86.11 \%)$. Fewer, but still the majority of patients, agreed that $\mathrm{OPaCT}$ provided them with new information $(61.11 \%)$ (Fig. 3).

Additionally, 34 participants (32 completers and two noncompleters) agreed to take part in the semi-structured interview at $T_{1}$. In the interviews, participants mentioned the following advantages of the web-based intervention: it could be administered flexibly in terms of time and place; it encouraged patients to engage with emotionally difficult issues and, simultaneously, it helped increase awareness of one's own strengths and resources. The feedback was perceived as highly personalized, and participants felt well understood. According to participants, the intervention could be improved by providing more in-depth information on specific topics, such as nutrition, fatigue, complementary medicine and on dealing with the palliative condition. Some participants missed the possibility of getting into contact with other patients, e.g. via chat, as well as an option of downloading and reading the individual lessons offline. It was also reported that due to fatigue or poor physical condition, the intervention was temporarily experienced as an additional strain, e.g. using the diaries was too time-consuming.

Table 1 Demographic and disease characteristics of participants and non-participants

\begin{tabular}{|c|c|c|c|c|c|}
\hline & & Total $(n=184)$ & Participants $(n=46)$ & Non-participants $(n=138)$ & $p$ \\
\hline \multicolumn{2}{|c|}{ Age in years (Mean, SD) } & $54.77(12.88)$ & $49.24(11.35)$ & $56.66(12.87)$ & 0.001 \\
\hline $\operatorname{Sex}(n, \%)$ & $\begin{array}{l}\text { Male } \\
\text { Female }\end{array}$ & $\begin{array}{l}77(41.85 \%) \\
107(58.15 \%)\end{array}$ & $\begin{array}{l}11(23.91 \%) \\
35(76.09 \%)\end{array}$ & $\begin{array}{l}66(47.83 \%) \\
72(52.17 \%)\end{array}$ & 0.005 \\
\hline \multirow[t]{7}{*}{ Cancer type $(n, \%)$} & $\begin{array}{l}\text { Breast } \\
\text { Female genitalia }\end{array}$ & $\begin{array}{l}68(36.96 \%) \\
16(8.70 \%)\end{array}$ & $\begin{array}{l}21(45.65 \%) \\
6(13.04 \%)\end{array}$ & $\begin{array}{l}47(34.06 \%) \\
10(7.25 \%)\end{array}$ & \multirow[t]{7}{*}{ N/A } \\
\hline & Male genitalia & $11(5.98 \%)$ & $5(10.87 \%)$ & $6(4.35 \%)$ & \\
\hline & $\begin{array}{l}\text { Pancreas } \\
\text { Stomach/oesophagus }\end{array}$ & $\begin{array}{l}18(9.79 \%) \\
17(9.24 \%)\end{array}$ & $9(19.57 \%)$ & $\begin{array}{l}9(6.52 \%) \\
17(12.32 \%)\end{array}$ & \\
\hline & Colon/rectum & $14(7.61 \%)$ & $5(10.87 \%)$ & $9(6.52 \%)$ & \\
\hline & Skin & $12(6.52 \%)$ & --- & $12(8.70 \%)$ & \\
\hline & Head and neck & $11(5.98 \%)$ & --- & $11(7.97 \%)$ & \\
\hline & Others & $17(9.24 \%)$ & --- & $17(12.32 \%)$ & \\
\hline Metastasised $(n, \%)$ & $\begin{array}{l}\text { Yes } \\
\text { No }\end{array}$ & $\begin{array}{l}58(31.52 \%) \\
126(68.48 \%)\end{array}$ & $\begin{array}{l}14(30.43 \%) \\
32(69.57 \%)\end{array}$ & $\begin{array}{l}44(31.88 \%) \\
94(68.12 \%)\end{array}$ & 0.856 \\
\hline
\end{tabular}

$p$ values from $\chi^{2}$ and independent samples $t$ test 
Fig. 3 Participant satisfaction with $\mathrm{OPaCT}$ intervention

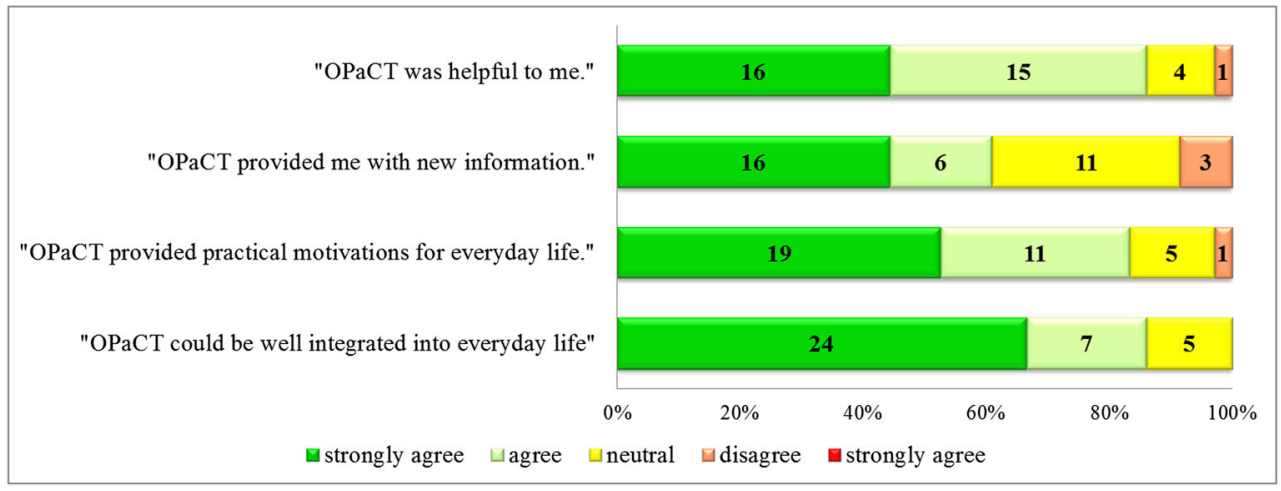

\section{Secondary Outcomes}

Based on 36 returned post-intervention questionnaires, a perprotocol analysis was conducted. Following correction with the Bonferroni-Holm method, the Wilcoxon signed-rank test indicated no improvement in depressive syndromes (PHQ-9: $z=-1.614, p=0.800)$, general anxiety disorder (GAD-7: $z=$ $-0.166, p=1.00$ ), overall supportive care needs (SCNSSF34-G: $z=-0.31, p=0.804)$ and self-efficacy for coping with cancer (CBI-B-D: $z=-1.147 p=1.00$ ) at the end of the intervention. Significant improvements from baseline $\left(T_{0}\right)$ to post-intervention $\left(T_{1}\right)$ were found in the SCNS-SF34 subscale 'psychological needs' $(z=-2.862, p=0.036)$ (see Table 2 ).

\section{Discussion}

In the present study, we investigated the feasibility and acceptability of a guided biopsychosocial online intervention for cancer patients undergoing chemotherapy $(\mathrm{OPaCT})$. The results of this feasibility study reveal initially a low uptake with a response rate of $25 \%$ but subsequently a high adherence and a completer rate of over $80 \%$. The post-treatment questionnaire indicated a high level of patient satisfaction with the intervention. The intervention was experienced as helpful, and it could be well integrated into everyday life. Participants showed significant improvement with regard to unmet psychological needs. The low uptake of OPaCT is in line with findings from other studies of IBIs. Ebert et al. [31] indicated that the uptake rates of IBIs for depression are currently rather low, varying between 3 and 25\%. Recent studies in cancer patients also encountered great difficulties regarding recruitment and a low participation rate in IBIs [14, 32]. In addition to the low uptake, two common reasons for non-participation were 'sense of overwhelming demands' and 'concerns about additional involvement with the disease'. This raises the question of the optimal time frame for intervention: should $\mathrm{OPaCT}$ occur as early as possible in order to optimally support patients during chemotherapy, or would it be more reasonable to offer the intervention in the course of chemotherapy if a
Table 2 PHQ-9, GAD-7, SCNSSF34 and CBI-B-D scores baseline $\left(T_{0}\right)$ and post-intervention $\left(T_{1}\right)$

\begin{tabular}{|c|c|c|c|c|c|c|c|}
\hline & \multicolumn{3}{|l|}{$T_{0}$} & \multicolumn{3}{|l|}{$T_{1}$} & \multirow[b]{2}{*}{$p$ value } \\
\hline & Med & Mean & SD & Med & Mean & SD & \\
\hline PHQ-9 score & 6.00 & 6.46 & 3.73 & 6.00 & 6.08 & 3.83 & .800 \\
\hline GAD-7 score & 5.00 & 5.28 & 3.67 & 4.00 & 5.29 & 3.79 & 1.00 \\
\hline SCNS-SF34 score & 71.00 & 75.89 & 26.45 & 69.00 & 66.52 & 17.75 & .804 \\
\hline \multicolumn{8}{|l|}{ SCNS-SF34 subscales: } \\
\hline Health system and information needs & 20.00 & 24.16 & 11.23 & 20.00 & 21.51 & 9.11 & 1.00 \\
\hline Psychological needs & 24.00 & 26.60 & 10.55 & 19.50 & 20.59 & 8.06 & .036 \\
\hline Physical and daily living needs & 9.00 & 9.72 & 3.92 & 9.00 & 9.46 & 2.66 & 1.00 \\
\hline Patient care and support needs & 7.50 & 8.78 & 4.24 & 7.00 & 7.42 & 2.71 & .800 \\
\hline Sexuality needs & 5.00 & 6.54 & 3.86 & 5.00 & 5.75 & 2.71 & 1.00 \\
\hline CBI-B-D score & 91.50 & 93.30 & 20.24 & 93.30 & 91.03 & 19.92 & 1.00 \\
\hline
\end{tabular}

$p$ values from Wilcoxon signed-rank test with Bonferroni-Holm correction.; Med, median; $P H Q-9$, depression module of the Patient-Health Questionnaire; GAD-7, anxiety module of the Patient Health Questionnaire; SCNS$S F 34-G$, German version of the Short-Form Supportive Care Needs Survey Questionnaire; $C B I-B-D$, German version of the brief form of the Cancer Behavior Inventory 
certain 'routine' with the treatment has been established? The low dropout rate of $19.6 \%$ and the high adherence rate of $80.4 \%$ in our study are noteworthy, especially because high attrition and low adherence are considered a main limitation of online self-help interventions in both cancer and non-cancer populations [33]. There is some evidence that an increased level of guidance or support leads to better adherence, and some studies emphasize the superiority of guided interventions over unguided interventions [34]. A core element of $\mathrm{OPaCT}$ is personal feedback, which the patients experienced as particularly supportive and beneficial. We therefore assume that the intensive and highly individualized personal guidance provided for the patients led to the high adherence and the low dropout rate.

About half of our participants were breast cancer patients, while patients with skin, stomach/oesophagus, or head and neck cancer were not represented. These results are consistent with findings that patient characteristics such as sex, age and tumour site are linked to the uptake of psycho-oncological support [35-37]. In our study sample, $15.2 \%$ of participants had a clinical indication for anxiety (GAD-7) and $21.8 \%$ for depression (PHQ-9). This is a lower percentage of highly distressed patients than in other studies reporting psychological distress of people newly diagnosed with cancer [1,2], but comparable with other studies on online-based interventions $[10,38]$. Comparing baseline scores of the completers and non-completers in our sample, however, we found that the PHQ-9 score at the beginning of the intervention was significantly higher for completers than non-completers. These results suggest that highly distressed patients, although they make less extensive use of $\mathrm{OPaCT}$, may particularly benefit from the intervention.

In recent years, a small number of IBIs were developed for patients undergoing chemotherapy, most of them focusing on management of chemotherapy-related symptoms or stress management [39]. The distinctive aspect of $\mathrm{OPaCT}$ is that the intervention is based on the Supportive Care Framework for Cancer Care [26] and focuses both on the physical side effects of chemotherapy and on emotional distress during treatment. The main strength of our study is that it is a biopsychosocial intervention developed on an interdisciplinary basis which addresses a broad group of patients. The intervention is patient oriented and can be used in clinical care independently of research. Another strength of this study is that we used a mixed-methods research design and collected multifaceted data regarding the feasibility and the acceptability of OPaCT. This enabled a good insight into which patients participated in the intervention and what the participating patients experienced as particularly helpful.

Some limitations of the study need to be acknowledged. Because feasibility and acceptability of the intervention were the main focus of our study, a single-arm study was conducted. There was no control group, and the sample size was small and not powered to detect changes in secondary outcome criteria. Although not essential for many aspects of this study, the inclusion of a control group would have allowed a more realistic examination of recruitment, randomization and implementation of the intervention. Our study sample in this pilot study was wide ranging: no specification was made for certain tumour types or for particularly distressed patients. The intervention should initially be made available to a large group of patients whose common denominator is the beginning of chemotherapy with its possible physical and psychological burdens. We accepted the resulting heterogeneity of our study sample to (1) prevent overlooking a small but important group of patients who might benefit from the intervention and (2) investigate whether there are specific patient groups (e.g. depending on cancer type) who use OPaCT in particular.

\section{Conclusion}

In summary, the guided biopsychosocial online intervention for cancer patients undergoing chemotherapy (OPaCT) has proven feasible and our results indicate that $\mathrm{OPaCT}$ can be implemented well both in the treatment process and in patients' everyday lives. The intervention has the potential to provide orientation and support in dealing with physical strains and emotional distress, and is aimed at reducing unmet supportive care needs of patients undergoing chemotherapy. The clinical effectiveness of OPaCT needs to be tested in an evaluative research design. This study showed that recruitment was more difficult than expected and that recruitment procedures should be carefully considered when planning a randomized controlled trial.

Acknowledgements We would like to thank Paula Hoffmann, Iris Aupperle, Katrin Willig, Catherine Schneider, Felix Berberich and the entire nursing team of the outpatient clinic of the National Center for Tumor Diseases in Heidelberg for their support in the conceptual design, development and realization of $\mathrm{OPaCT}$.

Authors' Contributions All authors made substantial contributions to the conception or design of the work, drafted the work or revised it critically for important intellectual content, approved the version to be published, and agreed to be accountable for all aspects of the work in ensuring that questions related to the accuracy or integrity of any part of the work are appropriately investigated and resolved.

Funding Information Open Access funding provided by Projekt DEAL. This work was supported by the NCT Elevator Pitch.

Data Availability The authors have full control of all primary data and agree to allow the journal to review all data if requested.

\section{Compliance with Ethical Standards}

Ethical Approval Ethical approval was granted by the Ethics Committee of the University of Heidelberg (S-320/2017). 
Conflict of Interest The authors declare that they have no conflicts of interest.

Consent to Participate Informed consent was obtained from all individual participants included in the study.

Open Access This article is licensed under a Creative Commons Attribution 4.0 International License, which permits use, sharing, adaptation, distribution and reproduction in any medium or format, as long as you give appropriate credit to the original author(s) and the source, provide a link to the Creative Commons licence, and indicate if changes were made. The images or other third party material in this article are included in the article's Creative Commons licence, unless indicated otherwise in a credit line to the material. If material is not included in the article's Creative Commons licence and your intended use is not permitted by statutory regulation or exceeds the permitted use, you will need to obtain permission directly from the copyright holder. To view a copy of this licence, visit http://creativecommons.org/licenses/by/4.0/.

\section{References}

1. Mehnert A, Brähler E, Faller H, Härter M, Keller M, Schulz H, Wegscheider K, Weis J, Boehncke A, Hund B, Reuter K (2014) Four-week prevalence of mental disorders in patients with cancer across major tumor entities. J Clin Oncol 32:3540-3546

2. Mehnert A, Hartung TJ, Friedrich M, Vehling S, Brähler E, Härter M, Keller M, Schulz H, Wegscheider K, Weis J, Koch U (2018) One in two cancer patients is significantly distressed: prevalence and indicators of distress. Psycho-Oncol 27:75-82

3. Fang F, Fall K, Mittleman MA, Sparén P, Ye W, Adami HO, Valdimarsdóttir U (2012) Suicide and cardiovascular death after a cancer diagnosis. N Engl J Med 366:1310-1318

4. Faller H, Schuler M, Richard M, Heckl U, Weis J, Kueffner R (2013) Effects of psycho-oncologic interventions on emotional distress and quality of life in adult patients with cancer: systematic review and meta-analysis. J Clin Oncol 31:782-793

5. Carlson LE, Angen M, Cullum J, Goodey E, Koopmans J, Lamont et al (2004) High levels of untreated distress and fatigue in cancer patients. Br J Cancer 90:2297-2304

6. Baker Glenn EA, Park B, Granger L, Symonds P, Mitchell AJ (2011) Desire for psychological support in cancer patients with depression or distress: validation of a simple help question. Psycho-Oncol 20:525-531

7. Leykin Y, Thekdi SM, Shumay DM, Muñoz RF, Riba M, Dunn LB (2012) Internet interventions for improving psychological wellbeing in psycho-oncology: review and recommendations. PsychoOncol 21:1016-1025

8. Agboola SO, Ju W, Elfiky A, Kvedar JC, Jethwani K (2015) The effect of technology-based interventions on pain, depression, and quality of life in patients with cancer: a systematic review of randomized controlled trials. J Med Internet Res 17:e65

9. McAlpine H, Joubert L, Martin-Sanchez F, Merolli M, Drummond KJ (2015) A systematic review of types and efficacy of online interventions for cancer patients. Patient Educ Couns 98:283-295

10. Willems RA, Mesters I, Lechner L, Kanera IM, Bolman CAW (2017) Long-term effectiveness and moderators of a web-based tailored intervention for cancer survivors on social and emotional functioning, depression, and fatigue: randomized controlled trial. J Cancer Surviv 11:691-703

11. David N, Schlenker P, Prudlo U, Larbig W (2013) Internet-based program for coping with cancer: a randomized controlled trial with hematologic cancer patients. Psycho-Oncol 22:1064-1072
12. Fergus KD, McLeod D, Carter W, Warner E, Gardner SL, Granek L, Cullen KI (2014) Development and pilot testing of an online intervention to support young couples' coping and adjustment to breast cancer. Eur J Cancer Care 23:481-492

13. Wootten AC, Abbott JAM, Meyer D, Chisholm K, Austin DW, Klein B, McCabe M, Murphy DG, Costello AJ (2015) Preliminary results of a randomised controlled trial of an online psychological intervention to reduce distress in men treated for localised prostate cancer. Eur Urol 68:471-479

14. Boele FW, Klein M, Verdonck-de Leeuw IM, Cuijpers P, Heimans JJ, Snijders TJ, Vos M, Bosma I, Tijssen CC, Reijneveld JC (2018) Internet-based guided self-help for glioma patients with depressive symptoms: a randomized controlled trial. J Neuro-Oncol 137:191203

15. Hummel SB, Van Lankveld JJ, Oldenburg HS, Hahn DE, Kieffer JM, Gerritsma MA, Lopes Cardozo AM (2017) Efficacy of internet-based cognitive behavioral therapy in improving sexual functioning of breast cancer survivors: results of a randomized controlled trial. J Clin Oncol 35:1328-1340

16. Zachariae R, Amidi A, Damholdt MF, Clausen CD, Dahlgaard J, Lord H, Ritterband LM (2018) Internet-delivered cognitive-behavioral therapy for insomnia in breast cancer survivors: a randomized controlled trial. J Natl Cancer Inst 110:880-887

17. Foster C, Grimmett C, May CM, Ewings S, Myall M, Hulme C, Breckons M (2016) A web-based intervention (RESTORE) to support self-management of cancer-related fatigue following primary cancer treatment: a multi-centre proof of concept randomised controlled trial. Support Care Cancer 24:2445-2453

18. Kearney N, McCann L, Norrie J, Taylor L, Gray P, McGee-Lennon M, Maguire R (2009) Evaluation of a mobile phone-based, advanced symptom management system (ASyMS@) in the management of chemotherapy-related toxicity. Support Care Cancer 17: $437-444$

19. Berry DL, Hong F, Halpenny B, Partridge AH, Fann JR, Wolpin S, Amtmann D (2014) Electronic self-report assessment for cancer and self-care support: results of a multicenter randomized trial. J Clin Oncol 32:199-205

20. Børøsund E, Cvancarova M, Moore SM, Ekstedt M, Ruland CM (2014) Comparing effects in regular practice of e-communication and web-based self-management support among breast cancer patients: preliminary results from a randomized controlled trial. J Med Internet Res 16:e295

21. Basch E, Deal AM, Kris MG, Scher HI, Hudis CA, Sabbatini P, Chou JF (2016) Symptom monitoring with patient-reported outcomes during routine cancer treatment: a randomized controlled trial. J Clin Oncol 34:557-565

22. Urech C, Grossert A, Alder J, Scherer S, Handschin B, Kasenda B, Gattlen L (2018) Web-based stress management for newly diagnosed patients with cancer (STREAM): a randomized, wait-list controlled intervention study. J Clin Oncol 36:780-788

23. Chircop D, Scerri J (2017) Coping with non-Hodgkin's lymphoma: a qualitative study of patient perceptions and supportive care needs whilst undergoing chemotherapy. Support Care Cancer 25:24292435

24. Sakamoto N, Takiguchi S, Komatsu H, Okuyama T, Nakaguchi T, Kubota Y, Akechi T (2017) Supportive care needs and psychological distress and/or quality of life in ambulatory advanced colorectal cancer patients receiving chemotherapy: a cross-sectional study. Jpn J Clin Oncol 47:1157-1161

25. Renovanz M, Hickmann AK, Coburger J, Kohlmann K, Janko M, Reuter AK, Giese A (2018) Assessing psychological and supportive care needs in glioma patients-feasibility study on the use of the Supportive Care Needs Survey Short Form (SCNS-SF 34-G) and the Supportive Care Needs Survey Screening Tool (SCNS-ST 9) in clinical practice. Eur J Cancer Care 27:e12598 
26. Fitch MI (2008) Supportive care framework. Can Onco Nurs J 18: 6-24

27. Spitzer RL, Kroenke K, Williams JB, Patient Health Questionnaire Primary Care Study Group (1999) Validation and utility of a selfreport version of PRIME-MD: the PHQ primary care study. Jama 282:1737-1744

28. Lehmann C, Koch U, Mehnert A (2012) Psychometric properties of the German version of the short-form supportive care needs survey questionnaire (SCNS-SF34-G). Support Care Cancer 20:24152424

29. Giesler J, Weis J (2008) Psychometric properties of the German brief form of the cancer behavior inventory. Psycho-Oncol 17: S174-S175

30. Holm S (1979) A simple sequentially rejective multiple test procedure. Scand J Stat 6:65-70

31. Ebert DD, Berking M, Cuijpers P, Lehr D, Pörtner M, Baumeister $H$ (2015) Increasing the acceptance of internet-based mental health interventions in primary care patients with depressive symptoms. A randomized controlled trial. J Affect Disord 176:9-17

32. Cockle-Hearne J, Barnett D, Hicks J, Simpson M, White I, Faithfull S (2018) A web-based intervention to reduce distress after prostate cancer treatment: development and feasibility of the getting down to coping program in two different clinical settings. JMIR cancer 4:e8

33. Donkin L, Christensen H, Naismith SL, Neal B, Hickie IB, Glozier N (2011) A systematic review of the impact of adherence on the effectiveness of e-therapies. J Med Internet Res 13:e52
34. Baumeister H, Reichler L, Munzinger M, Lin J (2014) The impact of guidance on internet-based mental health intervention-a systematic review. Internet Interv 1:205-215

35. Curry C, Cossich T, Matthews J, Beresford J, McLachlan S (2002) Uptake of psychosocial referrals in an outpatient cancer setting: improving service accessibility via the referral process. Support Care Cancer 10:549-555

36. Merckaert I, Libert Y, Messin S, Milani M, Slachmuylder JL, Razavi D (2010) Cancer patients' desire for psychological support: prevalence and implications for screening patients' psychological needs. Psycho-Oncol 19:141-149

37. Beatty L, Binnion C, Kemp E, Koczwara B (2017) A qualitative exploration of barriers and facilitators to adherence to an online selfhelp intervention for cancer-related distress. Support Care Cancer 25:2539-2548

38. Lee JY, Park HY, Jung D, Moon M, Keam B, Hahm BJ (2014) Effect of brief psychoeducation using a tablet PC on distress and quality of life in cancer patients undergoing chemotherapy: a pilot study. Psycho-Oncol 23:928-935

39. Moradian S, Voelker N, Brown C, Liu G, Howell D (2018) Effectiveness of Internet-based interventions in managing chemotherapy-related symptoms in patients with cancer: a systematic literature review. Support Care Cancer 26:361-374

Publisher's Note Springer Nature remains neutral with regard to jurisdictional claims in published maps and institutional affiliations. 